\title{
Determinación del pH y recuento de mesófilos en carne de boquichico (Prochilodus nigricans) proveniente de los mercados de la ciudad de Pucallpa, Ucayali
}

\author{
Determination of the $\mathrm{pH}$ and count of mesophilos in black prochilodus meat \\ (Prochilodus nigricans) from the markets of the city of Pucallpa, Ucayali \\ Nathaly Ponce P. ${ }^{1}$, Juan Rondón E. ${ }^{2,3}$, Manuel de la Torre V. ${ }^{1}$, \\ Yamili Mendoza Q. ${ }^{2}$
}

\section{Resumen}

\begin{abstract}
El objetivo del estudio fue determinar el $\mathrm{pH}$ y el recuento de mesófilos en carne de boquichico (Prochidolus nigricans), proveniente de los mercados de la ciudad de Pucallpa, Ucayali. Se recolectaron 115 muestras de ejemplares de boquichico de los 23 puestos de venta distribuidos en los 5 mercados de la ciudad ( 5 muestras por puesto, considerando al puesto de venta como un lote), mediante un muestreo no probabilístico por conveniencia. El procesamiento se realizó mediante la medición del $\mathrm{pH}$ de la carne y el análisis para el recuento de mesófilos con el método SPC (conteo de placas aeróbicas). Los promedios de los valores de $\mathrm{pH}$ en la carne de boquichico por mercado estuvieron por debajo del valor neutro $(\mathrm{pH}=7.0)$, pero fueron mayores en las muestras procedentes de los mercados 3 y 5; asimismo, los recuentos de mesófilos en la carne sobrepasaron a los Límites Máximos Permisibles en 4 de los 23 puestos de venta (dos del mercado N. ${ }^{\circ} 1$, uno del mercado $\mathrm{N}^{\circ} 2$ y uno del mercado $\mathrm{N} .^{\circ} 3$ ). Se concluye que la carne de boquichico comercializada en los mercados de la ciudad de Pucallpa no es apta para su consumo en tres puestos en dos mercados de Callería y en un puesto de un mercado en Manantay.
\end{abstract}

Palabras clave: boquichico, $\mathrm{pH}$, mesófilos, mercados, Pucallpa

${ }^{1}$ Facultad de Medicina Veterinaria, Universidad Alas Peruanas, filial Pucallpa, Perú

${ }^{2}$ Estación del Centro de Investigación IVITA Pucallpa, Facultad de Medicina Veterinaria, Universidad Nacional Mayor de San Marcos, Ucayali, Perú

${ }^{3}$ E-mail:jrondone@unmsm.edu.pe

Recibido: 19 de abril de 2020

Aceptado para publicación: 2 de enero de 2021

Publicado: 24 de abril de 2021 
The aim of this study was to determine the $\mathrm{pH}$ and the count of mesophiles in black prochilodus («boquichico») meat (Prochidolus nigricans), from the markets of the city of Pucallpa, Ucayali. In total, 115 samples of boquichico specimens were collected from the 23 stalls distributed in the 5 markets of the city ( 5 samples per stall, considering the stall as a lot), through a non-probabilistic convenience sampling. The processing was carried out by measuring the $\mathrm{pH}$ of the meat and the analysis for the count of mesophiles with the SPC method (aerobic plate count). The averages of the $\mathrm{pH}$ values in boquichico meat by market were below the neutral value $(\mathrm{pH}=7.0)$, but were higher in the samples from markets 3 and 5; likewise, the mesophilic counts in meat exceeded the Maximum Allowable Limits in 4 of the 23 stalls (two from market $\mathrm{N}^{\circ} 1$, one from market $\mathrm{N}^{\circ} 2$ and one from market $\mathrm{N}^{\circ} 3$ ). It is concluded that the boquichico meat commercialized in the markets of the city of Pucallpa is not suitable for consumption in three stalls in two Callería markets and in one stall in a market in Manantay.

Key words: black prochilodus, $\mathrm{pH}$, mesophiles, markets, Pucallpa

\section{INTRODUCCIÓN}

El consumo de productos hidrobiológicos provee de una de las principales fuentes de proteína y de divisas para la Amazonía Peruana, lo cual se traduce en un consumo per cápita anual de $15.4 \mathrm{~kg}$, indicador de su importancia para la alimentación de la población local (Produce, 2015). Entre las especies de mayor consumo en la ciudad de Pucallpa destaca el boquichico (Prochilodus nigricans), el bagre (Siluriforme) y la palometa (Mylossoma duriventre) (IMARPE, 2018).

Variaciones en el clima (temperatura y humedad) y las deficiencias en la aplicación de las normas sanitarias durante el expendio al consumidor pueden influir negativamente en la calidad del producto, generando alteraciones que pueden ir desde cambios inofensivos en las características organolépticas del alimento acelerando su descomposición, hasta consecuencias graves causadas por las Enfermedades Transmitidas por Alimentos (ETA) (Tecnología Pesquera, 2009; FAO, 2014).
El pescado es un producto muy vulnerable, pudiendo generar pérdidas económicas a los comerciantes y de alto riesgo para la salud de los consumidores, ya que tienen la posibilidad de actuar como vehículos transmisores de microorganismos patógenos (SANIPES, 2016), especialmente cuando son consumidos crudos o poco cocidos (Galán et al., 2008), lo que ha incrementado la preocupación del sector público por la inocuidad de estos alimentos.

Las deficiencias existentes en la cadena de comercialización pesquera incrementan el riesgo en la presentación de ETAs, repercutiendo negativamente en las actividades comerciales (SANIPES, 2016a). Dentro de las causales de pérdidas económicas se tienen los decomisos de productos en mal estado o que estando en buen estado son asociados con las ETA, así como la clausura de establecimientos que expenden alimentos, sean estos mercados o restaurantes (FAO/ OMS, 2015). Según la OMS (2015), el pH de la carne es un indicador físico del estado de frescura del pescado, importante para determinar la calidad de la carne del pescado. Por 
otro lado, las bacterias mesófilas aerobias tienen una tasa de crecimiento bastante elevada y su tiempo de generación es corto, siendo uno de los primeros indicadores del estado sanitario de la carne (Campuzano et al., 2015).

La falta de vigilancia de la calidad de los productos pesqueros en los mercados de la Amazonía demanda realizar monitoreos de calidad de los productos hidrobiológicos a fin de obtener información de línea base en la región. El objetivo de la presente investigación fue, por lo tanto, determinar el $\mathrm{pH}$ y realizar el recuento de mesófilos en carne de boquichico (Prochidolus nigricans), proveniente de los mercados de la ciudad de Pucallpa, Ucayali.

\section{Materiales y Métodos}

El estudio se realizó en la provincia de Coronel Portillo, departamento de Ucayali, Perú. Se recolectaron las muestras de los mercados ubicados en los distritos de Callería, Manantay y Yarinacocha de la ciudad de Pucallpa. El procesamiento se realizó en la Unidad de Diagnóstico en Laboratorio de la Sección de Sanidad Animal del Instituto Veterinario de Investigaciones Tropicales y de Altura (IVITA), sede Pucallpa, el cual es parte de la Facultad de Medicina Veterinaria de la Universidad Nacional Mayor de San Marcos. El estudio se realizó entre agosto de 2018 y marzo de 2019.

El muestreo fue no probabilístico por conveniencia. Se consideraron todos los puestos de venta de pescado distribuidos en los cinco mercados de la ciudad $(\mathrm{n}=23)$. Los mercados $\mathrm{N}^{\circ} 1$ y $\mathrm{N}^{\circ} 2$ en el distrito de Callería tuvieron ocho puestos, los mercados $\mathrm{N}^{\circ} 3 \mathrm{y}$ $\mathrm{N}^{\circ} 5$ ubicados en Manantay tuvieron nueve puestos y el mercado $\mathrm{N}^{\circ} 4$ en Yarinacocha tuvo seis puestos. En cada puesto de venta se recolectaron cinco ejemplares de boquichico (Prochilodus nigricans), obteniéndose en total 115 ejemplares.
La toma de muestra se realizó entre las 06:00 y 07:30 horas. Los peces fueron colocados en cajas térmicas con geles refrigerantes (entre 2 y $8{ }^{\circ} \mathrm{C}$ ) hasta su procesamiento dentro de las tres horas siguientes. El análisis microbiológico comprendió el recuento de mesófilos en Plate Count Agar (PCA) (Liofilchem $\left.{ }^{\circledR}\right)$. Se utilizó una porción de carne de la zona anterior del tronco sobre las costillas de cada ejemplar, siguiendo la metodología descrita por Da Silva et al. (2013). Los valores obtenidos en las muestras fueron comparados con la Norma Técnica Sanitaria otorgada por SANIPES (SANIPES, 2016b), referente a los límites máximos permisibles (LMP) para el recuento de mesófilos en carne de pescado. La medición de $\mathrm{pH}$ en la carne se hizo en la zona opuesta utilizada para el análisis microbiológico con un phmetro (Yaruifansen PH-013).

Se aplicó una estadística descriptiva mediante diagrama de cajas representando el promedio y la mediana de los valores de $\mathrm{pH}$ y el recuento de mesófilos hallados en los lotes muestreados de cada mercado. Se utilizó la prueba de Kruskal-Wallis para determinar la diferencia de las medianas de los valores entre mercados con un nivel de significancia de 0.01 y la prueba de Dunn para determinar la diferencia significativa en la mediana entre uno y otro grupo de muestras de los lotes por mercado mediante comparaciones múltiples, con un nivel de significancia de 0.05 . Finalmente, se realizó un diagrama de dispersión para los valores de $\mathrm{pH}$ y el recuento de mesófilos en las muestras de los cinco mercados. Los resultados fueron esquematizados con el programa Sigmaplot 11.0.

\section{Resultados}

En la Figura 1, el diagrama de cajas representa los valores de $\mathrm{pH}$ obtenidos de las muestras de carne de boquichico de los cinco mercados de Pucallpa. Todos los promedios y medianas estuvieron por debajo del $\mathrm{pH}$ neutro ( $\mathrm{pH} 7$ ). Las muestras de los merca- 


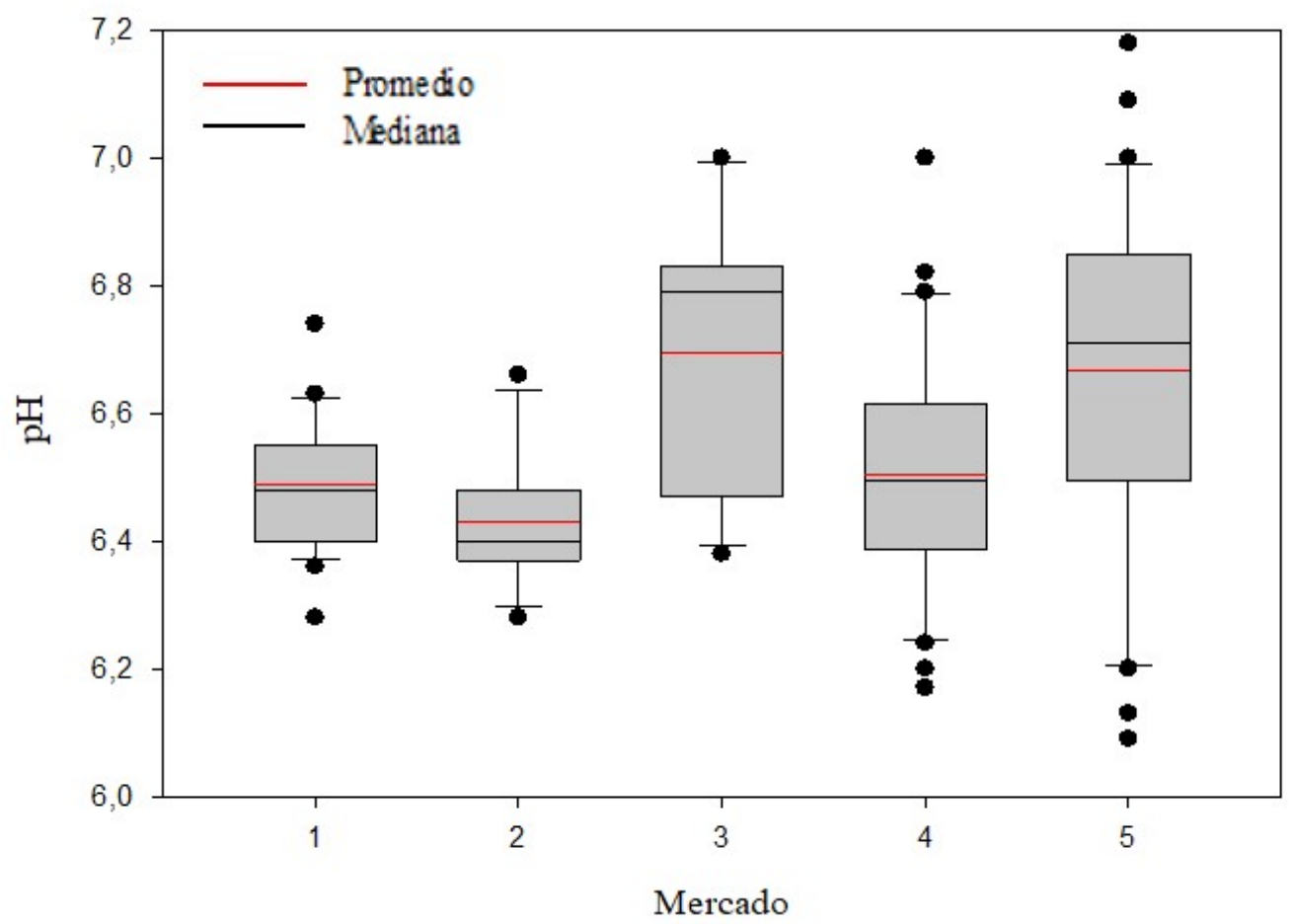

Figura 1. Diagrama de cajas de los valores de $\mathrm{pH}$ en carne de boquichico (Prochilodus nigricans) comercializado en cinco mercados de Pucallpa, Ucayali (2018-2019)

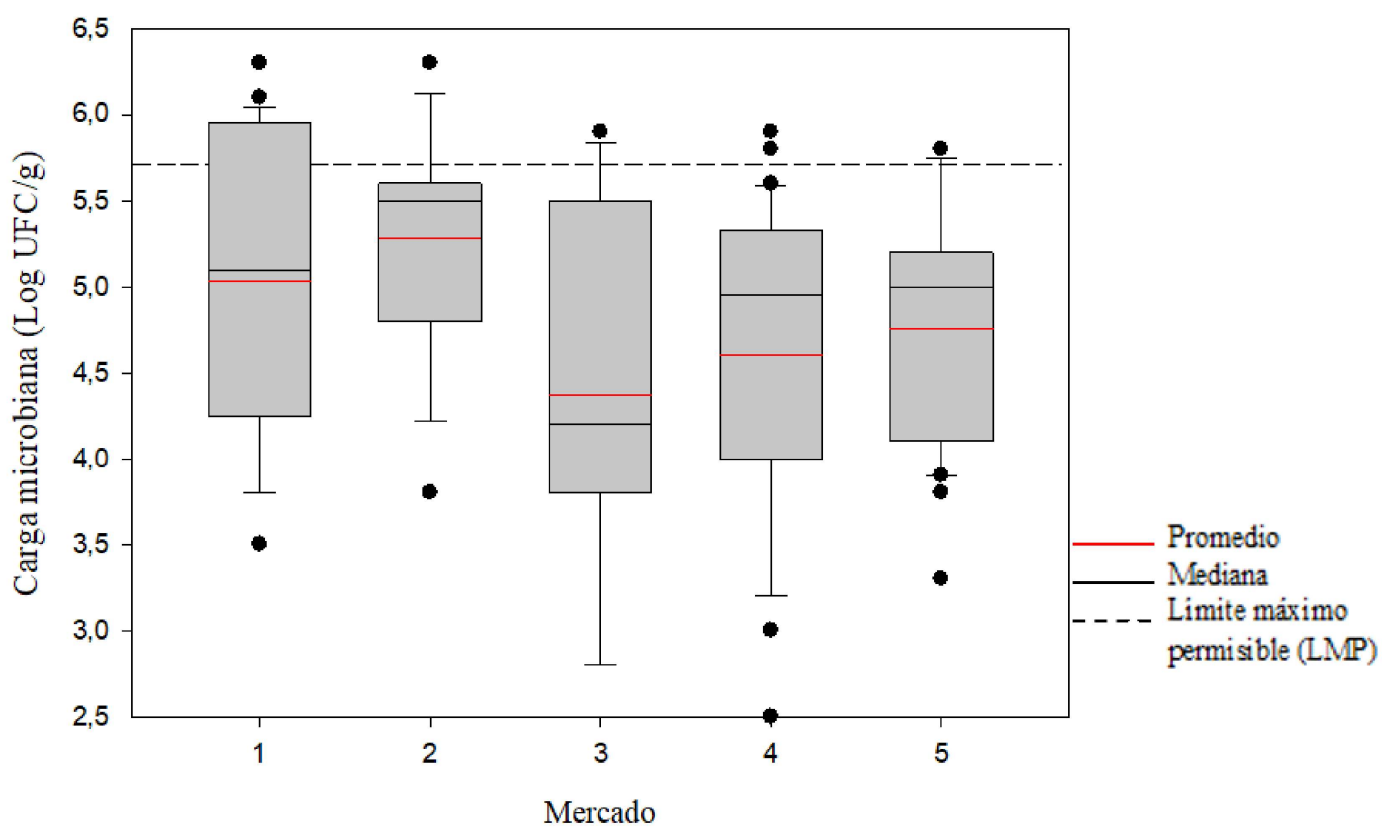

Figura 2. Diagrama de cajas de los resultados en el recuento de bacterias mesófilas en carne de boquichico (Prochilodus nigricans) (Log UFC/g) comercializada en cinco mercados de Pucallpa, Ucayali (2018-2019) 


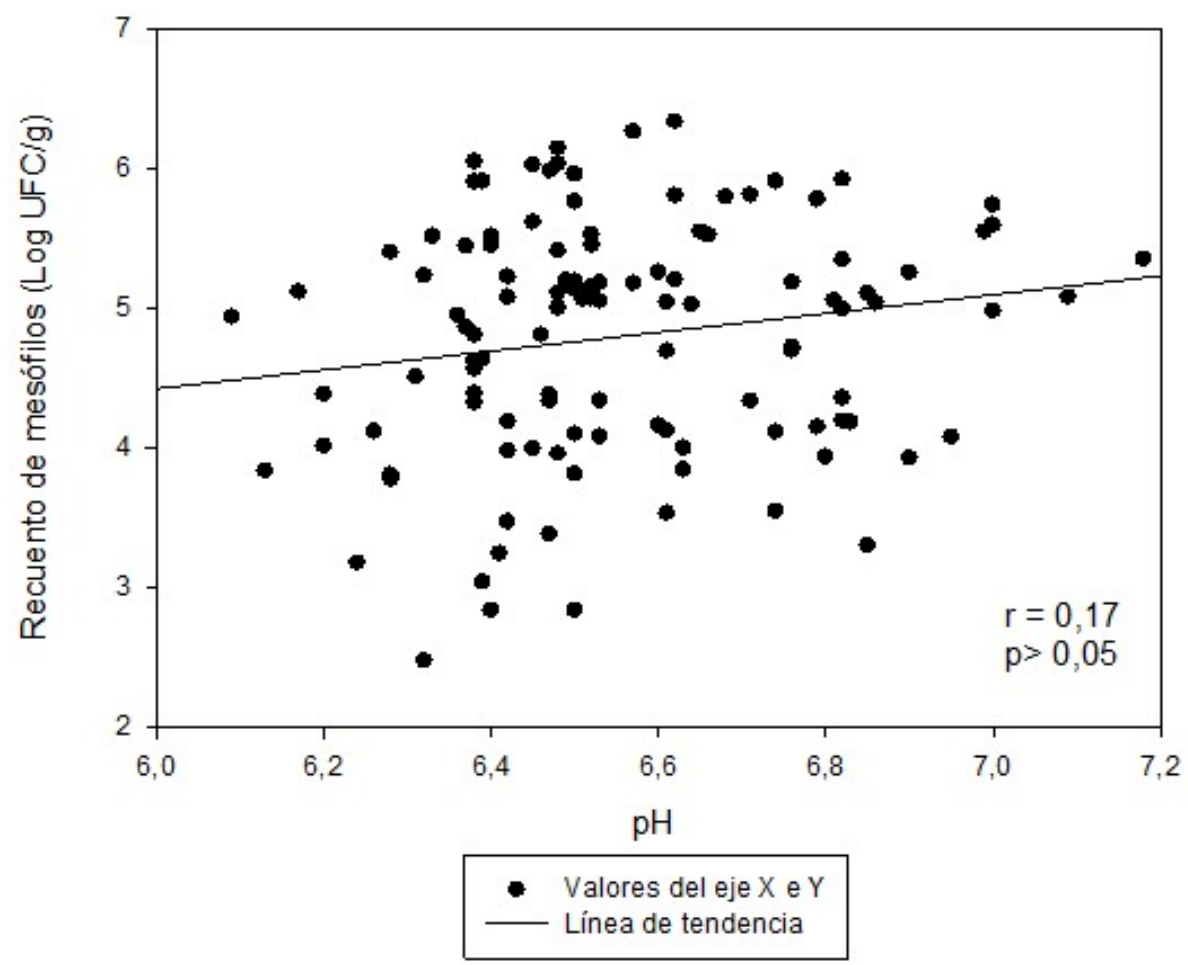

Figura 3. Diagrama de dispersión entre los valores de $\mathrm{pH}$ y el recuento de mesófilos en la carne de boquichico recolectada en cinco mercados de abastos de Pucallpa, Ucayali (2018-2019)

dos 1,2 y 4 , tuvieron rangos intercuartiles cortos y los promedios de $\mathrm{pH}$ fueron 6.49 , 6.42 y 6.50 , respectivamente, mayores a sus medianas. De otra parte, hubo una mayor variación en los valores extremos de las muestras en el mercado 4, llegando a valores de 7. En los mercados 3 y 5, los rangos intercuartiles fueron amplios, los promedios de $\mathrm{pH}$ fueron 6.69 y 6.50 , respectivamente, menores a sus medianas, y con mayor cantidad de valores extremos en los grupos de muestras del mercado 5 (por debajo de 6.2 y sobre 7).

La prueba de Kruskal-Wallis indica diferencias significativas para los valores de $\mathrm{pH}$ de las muestras entre mercados $(\mathrm{p}<0.01)$ y la prueba de Dunn determinó diferencias significativas $(p<0.05)$ entre los valores de $\mathrm{pH}$ de los mercados 2 y 3 , así como entre los mercados 2 y 5 .
En la Figura 2, el diagrama de cajas representa los valores del recuento de mesófilos obtenidos de la carne de boquichico en las muestras de los cinco mercados de Pucallpa. Los promedios y medianas en el total de las muestras tuvieron cargas por debajo a los valores Límites Máximos Permisibles (LMP). Los rangos intercuartiles fueron más amplios en las muestras de los mercados 1 y 3 , con valores extremos más distantes en este último, a diferencia de los demás mercados; asimismo, los valores promedio fueron más bajos en las muestras de los mercados 3, 4 y 5. Por otra parte, hubo valores de muestras mayores a 5.69 Log UFC/g en todos los mercados; sin embargo, al cotejarse con la Norma Sanitaria, la carne no fue apta solo en cuatro puestos de los mercados 1,2 y 3 .

La prueba de Kruskal-Wallis indica diferencias significativas en el recuento de bac- 
terias mesófilas en carne de boquichico entre mercados $(\mathrm{p}=0.028)$ y la prueba de Dunn determinó diferencia significativa $(\mathrm{p}<0.05)$ entre los valores de los recuentos de los mercados 2 y 3 .

El coeficiente de correlación de Pearson entre el $\mathrm{pH}$ de la carne y el recuento total de bacterias mesófilas aerobias fue de $\mathrm{r}=0.172$, sin que fuera estadísticamente significativa $(\mathrm{p}=0.0668)$, observándose una marcada dispersión de los valores calculados de la línea de tendencia del gráfico (Figura 3).

\section{Discusión}

La presencia de microorganismos mesófilos en muestras cárnicas incluye tanto a microorganismos patógenos como no patógenos; además, refleja las condiciones de manipulación, el estado de frescura o descomposición y la calidad sanitaria de los alimentos (Navarro, 2107). De otra parte, la medición del $\mathrm{pH}$ permite conocer el estado de frescura, de allí que ambos parámetros podrían determinar la calidad de la carne (FAO, 1998). No obstante, el recuento de mesófilos es el que determina la aceptación o el rechazo de los lotes (SANIPES, 2016), y este rechazo podría deberse a un alto recuento, producto de la contaminación cruzada en la manipulación y a las deficientes condiciones de conservación en cada puesto de venta, lo que no necesariamente tiene relación con el deterioro y la vida útil de la carne.

La importancia de evaluar la calidad de la carne en el boquichico se debe a que es una de las más comercializadas en la región (IMARPE, 2018), especie que al igual que otras especies amazónicas, tiene una alta probabilidad de contaminarse después de su extracción y durante la manipulación (Pao et al., 2008); asimismo, es importante conocer la calidad con la que llega a los mercados «secundarios», que corresponden al último eslabón de la cadena de comercialización pesquera ante el consumidor local.
Aunque los promedios de valores de $\mathrm{pH}$ fueron cercanos a 6.5 en todos los mercados (Figuras 1 y 2), según Tecnología Pesquera (2009), estos valores podrían favorecer el crecimiento exponencial de microorganismos si no se maneja la cadena de frío. No obstante, estos valores de $\mathrm{pH}$ se encuentran dentro de los rangos referidos por la PAHO como usuales para productos hidrobiológicos (PAHO, 2020). Cabe señalar que el pH encontrado en el presente estudio fue menor a los valores reportados por Rondón et al. (2020) en los comercializadores primarios de pescado ubicados en el puerto de Pucallpa, lo que sugiere que la carne en los mercados posiblemente tenga un menor estado de frescura.

Los recuentos de aerobios mesófilos estuvieron por encima a los LMP en la carne procedente de algunos lotes de muestras de los mercados 1 y 2 en Callería y del mercado 3 en Manantay (Figura 2), indicando su inaceptabilidad para el consumo. De acuerdo con Corrales et al. (2011), el proceso de cortado del pescado antes de su venta incrementaría la probabilidad de contaminación cruzada por contacto directo con el manipulador, las superficies y los utensilios empleados. Por otro lado, la falta de cadena de frío y el incremento de $\mathrm{pH}$ promoverían el crecimiento bacteriano en la carne.

Diversos aerobios mesófilos, incluidos los patógenos, que pudieran estas presentes en la carne del boquichico pueden ser destruidos mediante la cocción; no obstante, la tradición local del consumo de pescado crudo, ligeramente cocido o marinado pueden conllevar a que agentes infecciosos como Escherichia coli, Salmonella sp, o toxigénicos como Staphylococcus aureus puedan ser transferidos directamente por el hombre cuando la manipulación de los pescados es inadecuada (Da Silva et al., 2008).

Los resultados de los análisis microbiológicos en la carne de pescado se contrastan a los hallazgos reportados por Gabancho 
(2014), quien encontró una baja calidad higiénica en cuatro especies comercializadas en un mercado de la ciudad de Tingo María; así como con el reporte de Vásquez et al. (2018), quienes determinaron que los niveles de UFC/g de E. coli y $S$. aureus superaron los niveles permitidos por la norma técnica en varios mercados y mercadillos de la ciudad de Huánuco; y el trabajo de Ninahuamán (2019), quien encontró una baja calidad sanitaria del jurel comercializado en mercados de la ciudad de Arequipa, todos estos en Perú. No obstante, los niveles de contaminación encontrados en el presente estudio no fueron muy elevados, considerando que la calidad sanitaria de la carne de boquichico fue inaceptable en solo 4 de los 23 puestos de venta (en tres de los cinco mercados evaluados).

\section{Conclusiones}

- Los valores promedio de $\mathrm{pH}$ en la carne de boquichico (Prochidolus nigricans) en cada uno de los cinco mercados de la ciudad de Pucallpa estuvieron por debajo del valor neutro.

- Los recuentos de mesófilos en la carne sobrepasaron a los límites máximos permisibles (LMP) en 4 de los 23 puestos de venta (en tres de los cinco mercados).

- La carne de boquichico no se encontró apta para el consumo en tres puestos en dos mercados de Callería y en un puesto de un mercado en Manantay.

\section{Literatura Citada}

1. Campuzano S, Mejía D, Madero C, Pabón P. 2015. Determinación de la calidad microbiológica y sanitaria de alimentos preparados vendidos en la vía pública de la ciudad de Bogotá D.C. NOVA 13: 81-92. doi: 10.22490/ 24629448.1708

2. Corrales L, Alvarado M, Castillo L, Camacho Y. 2011. Estudio bacteriológico de la calidad del pescado fresco, bagre (Pseudoplatystoma sp) y mojarra roja (Oreochromis sp) comercializado en el municipio de El Colegio, Cundinamarca (Colombia). NOVA 9: 149-157. doi: 10.22490/24629448.497

3. Da Silva ML, Matté GR, Leal PM, Matté MH. 2008. Occurrence of pathogenic microorganisms in fish sold in Sao Paulo, Brazil. J Food Safety 30: 94-110. doi: 10.1111/j.1745-4565.2009.-00192x

4. Da Silva N, Hiromi M, Amstalden J, Ferraz De Arruda N, Da Silva M, Romeiro R. 2013. Microbiological examination methods of food and water. London: $484 \mathrm{p}$.

5. [FAO] Organización de las Naciones Unidas para la Agricultura y la Alimentación. 1998. El pescado fresco: su calidad y cambios en su calidad. [Internet]. Disponible en: http:// www.fao.org/3/v7180s/v7180s00.htm

6. [FAO] Food and Agriculture Organization of the United Nations. 2014. El estado mundial de la pesca y la acuicultura. Roma: FAO. [Internet]. Disponible en: http://www.fao.org/3/ca9229es/ca9229es.pdf

7. [FAO/OMS] Organización de las Naciones Unidas para la Agricultura y la Alimentación / Organización Mundial de la Salud. 2015. Norma general para los contaminantes y las toxinas presentes en los alimentos y piensos. Codex Alimentarius. Normas internacionales de los alimentos. CODEX STAN 193-2015. Enmienda 2010, 2012,2013,2104, 2015.62p.

8. Galán L, Luna Olvera H, García J. 2008. Control de calidad de productos pesqueros. Acta Pesquera 1: 52-66.

9. Gabancho TF. 2014. Evaluación de la calidad higiénica de 4 especies de pescado de mayor consumo, expendidos en el mercado de Tingo María. Tesis de Ingeniero Zootecnista. Tingo María, Perú: Univ. Nacional Agraria de la Selva. $139 \mathrm{p}$.

10. [IMARPE] Instituto del Mar del Perú. 2018. Resumen mensual de la pesquería comercial en Pucallpa - Ucayali. Seguimiento Biológico. [Internet]. Disponible en: http://www.imarpe.pe/imarpe/ 
archivos/reportes/imarpe_repor_noviembre2018_ucayali.pdf

11. Navarro J. 2017. Evaluación de la calidad microbiológica de Trachurus picturatus murphyi «jurel» y Aulacomya ater «choro» comercializados en diferentes mercados de los distritos de San Juan de Lurigancho y San Martín de Porres, Lima - Perú. Tesis de Bióloga Microbióloga Parasitóloga. Lima: Univ. Nacional Mayor de San Marcos. 100 p.

12. Ninahuamán E. 2019. Evaluación de la calidad bacteriológica de Trachurus picturatus murphyi «Jurel» expendido en los diferentes mercados de la Plataforma comercial Andrés Avelino Cáceres en la ciudad de Arequipa, 2019. Tesis de Biólogo. Arequipa: Univ. Nac San Agustin de Arequipa. 100 p.

13. [OMS] Organización Mundial de la Salud. 2015. Inocuidad de los alimentos. Nota descriptiva $\mathrm{N}^{\circ} 399$. [Internet]. Disponible en: http://www.who.int/ mediacentre/factsheets/fs399/es/

14. [PAHO] Pan American Health Organization. 2020. Peligros biológicos. Inocuidad de alimentos - Control Sanitario-HACCP. [Internet] Disponible en: http://www.paho.org/hq/index.php?option $=$ com_content $\&$ view $=$ article\&id=10838:2015-peligrosbiologicos\&Itemid $=41432$ \&lang $=$ en

15. Pao S, Ettinger M, Khalid M, Reid A, Nerrie B. 2008. Microbial quality of raw aquacultured fish fillets procured from Internet and local retail markets. J Food Protect 71: 1544-1549. doi: 10.4315/ 0362-028X-71.8.1544

16. [PRODUCE] Ministerio de la Producción. 2015. Consumo per-cápita de pescado a nivel nacional. [Internet]. Disponible en: https://gestion.pe/economia/produce-consumo-per-capita-pescado-nivel-nacional-crecio-10-12-3-kilos132287-noticia/
17. Rondón J, Ramos D, Vilca M, Salazar E, Mendoza Y, González R. 2020. Caracterización sanitaria e identificación de los puntos de contaminación microbiológica en la cadena de comercialización pesquera en el puerto de Pucallpa, Ucayali, Perú. Rev Inv Vet Perú 31: e17539. doi: 10.15381/rivep.v31i1.17539

18. [SANIPES] Organismo Nacional de Sanidad Pesquera. 2016a. Lineamiento para el expendio de pescados, mariscos y/o productos hidrobiológicos en mercados de abasto mayoristas y minoristas. RD $\mathrm{N}^{\circ}$ 017-2016-SANIPESDSNPA. [Internet] Disponible en: http:/ /www.sanipes.gob.pe/procedimientos/ 8_RESOLUCION_DIRECTORAL_N_017_2016_SANIPES_DSNPA.pdf

19. [SANIPES] Organismo Nacional de Sanidad Pesquera. 2016b. Manual «Indicadores Sanitarios y de Inocuidad para los Productos Pesqueros y Acuícolas para Mercado Nacional y de Exportación». [Internet] Disponible en: https:// cdn.www.gob.pe/uploads/document/file/ 435258/-137669951669343547320191203-9067-q8ay4m.pdf

20. [SENHAMI] Servicio Nacional de Meteorología e Hidrología del Perú. 2017. Clima. Mapa Climático del Perú. [Internet]. Disponible en: https:// www.senamhi.gob.pe/?p=mapa climatico-del-peru

21. Tecnología Pesquera. 2009. Microbiología de los alimentos: microbiología de pescados y mariscos. Biblioteca de la Universidad Nacional de Santa. [Internet]. Disponible en: https://es.scribd.com/ doc/22280512/Microbiologia-de-Pescados-y-Mariscos

22. Vásquez JM, Tasayco WR, Chuquiyauri MA, Apac S. 2018. Evaluación microbiológica de pescados y mariscos expendidos en mercados de la ciudad de Huánuco. Rev Inv Valdizana 12: 75-82. doi: 10.33554/riv.12.2.142 